\title{
Cost and quality of diabetes care: comparisons between Rzeszow, Podkarpacie, Poland and Waukesha, Wisconsin, United States
}

\section{ABSTRACT}

Background. The cost of diabetes care increases worldwide and is highest in the United States (US), while the quality of care remains unsatisfactory.

The aim of this study was to compare the quality and cost of type 2 diabetes mellitus (DM) care between Waukesha, Wisconsin, US and Rzeszów, Poland.

Methods. DM quality data for the Polish cohort were abstracted from the charts of 79 DM patients in Rzeszow, Podkarpacie from 1 January 2013 to 31 December 2014. Cost data were attained from the Polish National Health Fund. Seventy-nine DM patients, matched for age, body mass index, and sex, from Waukesha, Wisconsin were chosen as comparators. DM quality data was obtained from the medical record and cost data from health system decision support staff.

Results. Average $\mathrm{HbA}_{1 \mathrm{c}}(\%$, mean $\pm \mathrm{SD})$ in the Polish and US cohorts were $7.4 \pm 1.4$ and $8.0 \pm 2.1$, respectively $(P=0.03)$. Mean systolic/diastolic blood pressure $(\mathrm{mm} \mathrm{Hg})$ in the two cohorts was $150 \pm 17 / 81 \pm 12$ and $132 \pm 17 / 74 \pm 11(P<0.001)$, respectively. The rates of statin usage were $90 \%$ and $86 \%(P=0.45)$, respec-

Address for correspondence:

Mariusz Dąbrowski

NZOZ Beta-Med

Plac Wolności 17, 35-073 Rzeszów, Poland

Phone: +48178591560

Fax: +48178591561

Mobile: +48604 211824

e-mail: mariusz.dabrowski58@gmail.com

mdabrowski@univ.rzeszow.pl

Clinical Diabetology 2020, 9; 6: 469-474

DOI: $10.5603 /$ DK.2020.0051

Received: 05.08.2020

Accepted: 03.11.2020 tively. Costs of direct medical care (hospitalizations, outpatient care, and medications) in the Polish and US cohorts were 1,263 US dollars (USD) and 10,121 USD, per annum, respectively.

Conclusion. This study reports significant differences in cost with relatively small differences in quality and of DM care between Poland and the US. As the US continues to attempt healthcare reform in order to decrease cost and increase quality, this study suggests that gains in cost and quality may not be mutually exclusive. (Clin Diabetol 2020; 9; 6: 469-474)

Key words: diabetes, the cost of care, quality of care, Poland, United States

\section{Introduction}

Healthcare costs in the United States (US) are the highest in the world and are rising [1, 2]. In contrast, healthcare spending is much lower in European countries; e.g., 9,892 US dollars (USD) per capita in the US versus 6,647 USD per capita in Norway in 2016 which has the fourth-highest healthcare spending per capita in the world [3]. Other countries in Europe, such as Poland, have even lower healthcare costs [3]. According to the Organization for Economic Cooperation and Development (OECD), Poland spent just $6.4 \%$ of its gross domestic product (GDP) on healthcare in 2013, while the US spent $16.9 \%$ of its GDP on healthcare that same year. Despite the higher cost of healthcare in the US, life expectancies at birth were similar: 77.7 years in Poland and 78.8 years in the US for a person born in the same year [4]. In the healthcare cost debate occurring in the US, there are perhaps lessons to be learned from the global community [5]. 
Chronic diseases like type 2 diabetes mellitus (DM) account for a significant proportion of the total money spent on healthcare in the US [6]. Patients with DM account for 1 in 4 healthcare dollars spent in the US, with nearly half of that expenditure related directly to DM [6]. Previous studies estimated that the direct medical cost per patient with DM in the US was 10,000 USD/ year in 2017 [6, 7]. In other middle- and high-income countries, costs are significantly lower [8-10]. One of the lingering questions in the healthcare cost debate is whether the amount of money spent correlates with the quality of care $[2,11,12]$. Life expectancy data noted above seem to indicate that cost and quality may not always correlate; however, there are no data directly comparing the quality and the cost of DM care between the US and a middle-income country such as Poland.

Any analysis comparing the health systems of the two countries must control for potential variables that may confound the conclusion [13]. Significant potential confounders include patient-level demographics that would affect the cost or quality of care in a way that is unrelated to the health system. Other potential confounders include socioeconomic status and race.

The goal of this study is to compare the quality of care and approximate costs for DM care between two cities in the US and Poland Waukesha, US and Rzeszow, Poland while controlling for potential confounders. The study will seek to answer whether an increase in the cost of care leads to a commensurate increase in quality of care.

\section{Methods}

Subjects

Charts from seventy-nine patients with DM attending a Diabetic Outpatient Clinic of the second level of reference in Rzeszow, Poland (regional capital of Podkarpacie region of Poland) during the years 2013-2014 were randomly selected and quality of care data were collected. An equal number of patients (with a mailing address in the city of Waukesha, WI, US) attending an Endocrinology Clinic in the Froedtert Health system (State of Wisconsin) were reviewed and quality of care data was similarly extracted. Charts in both cohorts were only included if patients were aged $\geq 18$ years, had an established DM diagnosis, had documented glycated hemoglobin $\left(\mathrm{HbA}_{1 c}\right)$ values during the years of review, and an up-to-date medication list. Patients had to have received care (an office visit) at the above clinics at least once during the years 2013-2014. Both groups were cared for by board-certified Diabetologist or Endocrinologists.

Subjects in the two cohorts were matched for gender, the decade of life, and the World Health Or- ganization (WHO) body mass index (BMI) category. The Polish cohort was the basis for selecting matched patients in the Wisconsin cohort. The initial Wisconsin cohort was identified using a database extracted from the health system's electronic medical record (EMR) database and resulted in an initial cohort of $\sim 5000$ patients. Subsequently, the cohort was narrowed down to those who matched on WHO BMI category, gender, and the decade of life. Froedtert Health system consists of several large hospitals and over one million outpatient visits each year [14]. Institutional Review Board approval was obtained from both Rzeszow University in Rzeszow, Poland and the Medical College of Wisconsin in Milwaukee, Wisconsin.

\section{Regions of comparison}

The cities in this study were selected as comparators due to similarities in size, racial makeup, median income, and unemployment rate. Rzeszow is a city with a population of 188,606 in 2016 , nearly $100 \%$ white, a median income of 28,847 USD in 2015 , and an unemployment rate of $7.3 \%$ in 2015 [15]. Waukesha, WI is a city with a population of 72,363 in $2016,86 \%$ white, a median income of 31,874 USD in 2015, and an unemployment rate of $6.3 \%$ in 2015 [16]. Waukesha, WI was the closest in socioeconomic indicators to Rzeszow, Podkarpacie among the surrounding cities served by Froedtert Health system and was therefore used as a comparison city. The income levels were normalized based on Purchasing Power Parity (PPP) 2015 Polish zloty (PLN) and 2015 US dollars (USD) of 1.76 PLN per USD [17]. PPP normalizes for both the exchange rate and local purchasing power of each currency [18].

\section{Quality of DM care}

Quality metrics were selected from the American Diabetes Association (ADA) clinical guidelines [19]. The quality measures that were collected for both Rzeszow and Waukesha included $\mathrm{HbA}_{1 c^{\prime}}$ blood pressure (BP), rates of statin usage, nephropathy, and retinopathy. Rate of statin usage is defined as a prescribed statin in the chart or a documented statin allergy. Nephropathy is defined as a lab test positive for proteinuria or documentation of nephropathy in the provider note. Retinopathy is defined as documentation of such by an ophthalmologist on a dilated eye exam. These data were collected through chart reviews in Poland. Data for the WI cohort were collected through the EMR database $\left(\mathrm{HbA}_{1 \mathrm{c}}\right.$ and $\left.\mathrm{BP}\right)$, and the remainder (statin usage, nephropathy and retinopathy) were obtained through chart review.

$\mathrm{HbA}_{1 \mathrm{c}}$ and albuminuria measurements were performed using a DCA $2000^{\circledR}+$ analyzer (Siemens, 
Elkhart, IN, USA) using the monoclonal antibody method in Poland. In the US cohort, $\mathrm{HbA}_{1 \mathrm{c}}$ measurements were performed using the same DCA $2000^{\circledR}+$ analyzer while albuminuria assessments were performed using a Roche Cobas 8000 (Roche Diagnostics, Mannheim, Germany).

\section{Cost of DM care}

The cost of care data for the Polish cohort was provided by the Polish National Health Fund (NFZ), which is a Polish government entity that pays for the vast majority of care in Poland [20]. The NFZ provided data in aggregate for the region of Podkarpacie. Cost data included the cost of visits granted by NFZ in outpatient DM clinics, hospitalizations of patients with DM (including DM as comorbidity), and reimbursement of DM medications for patients with DM in the Podkarpacie region during years 2013-2014. The NFZ reported the number of unique patients with $D M$ included in the cost data. The cost data was converted into USD using PPP for the year in which the service was given. The total direct medical cost was calculated by adding together the costs of medications, outpatient visits, and inpatient care. The cost calculated in this study is an average for patients with DM living in Podkarpacie, Poland.

The cost of care for the Waukesha cohort was calculated directly from the hospital and professional billing records captured in the Froedtert Health system. Cost is reported as the direct medical cost during the years 2013-2014 at the estimated Medicare reimbursement, regardless of patient's insurance coverage, for a given service as calculated by Froedtert Health system decision support staff. Direct medical cost includes the costs of medications, outpatient visits, and inpatient care. The cost of medications only includes data from pharmacies operated by the health system. Additionally, the cost is reported as a percentage of gross national income (GNI) in 2013 as reported by the OECD for both Poland and the US.

\section{Data analyses}

All data were extracted into an Excel spreadsheet. Descriptive statistics (mean, standard deviation, sample proportions, etc.) were used to describe the distribution of quality variables and complications within the study sample. Continuous variables are presented as the mean (standard deviation). Two-tailed t-tests were used to test for differences across groups for continuous variables and a chi-square test was used for categorical variables. Significance level was set at $\mathrm{P}<0.008$ to account for multiple comparisons $(0.05 / 6)$ using Bonferroni correction [21].

\section{Results}

Quality of care data was collected from 158 patients ( $43 \%$ women in both cohorts) with DM in Podkarpacie and Wisconsin. Mean ages of patients from Poland and the US cohorts were 63.6 (9.0) and 63.3 (9.7) years $(P=.83)$, respectively. Mean BMI was 31.9 (4.7) and $32.4(5.0) \mathrm{kg} / \mathrm{m}^{2}(P=.57)$ in the Polish and US cohorts, respectively.

\section{Comparison of quality of care}

$\mathrm{HbA}_{1 \mathrm{c}}$ level and nephropathy rate were significantly lower in Polish cohort, while both systolic and diastolic $B P$ values were lower in the US patients. Statin use and retinopathy prevalence were not significantly different (Table 1). In the Polish and US cohorts, $84 \%$ and $99 \%$, respectively, were screened for nephropathy, while $100 \%$ and $85 \%$, respectively, were tested for diabetic retinopathy.

\section{Comparison of costs}

The mean direct cost of care (cost of hospitalizations, outpatient care, and medications) per patient expressed in USD and as \% of the GNI per capita was considerably higher in the US compared to Poland (Fig. 1).

\section{Discussion}

In this study of two cohorts from comparable cities in Poland and the US, we found that glycemic control as measured by $\mathrm{HbA}_{1 \mathrm{c}}$ was not statistically different between the two groups, but there was a significant difference in direct care costs. BP levels were statistically significantly lower in the US cohort compared to the Polish cohort. Nephropathy rates were higher in the US cohort although fewer patients were evaluated for nephropathy in the Polish cohort. Retinopathy rates and statin usage were not significantly different between the two groups. This is the first study, of which we are aware, to compare the quality of care in cohorts between two countries with different healthcare systems in matched cohorts.

In this study, we chose objective measures to assess the quality of DM care. American Diabetes Association guidelines recommend measurement of $\mathrm{HbA}_{1 \mathrm{c}}$ every 3-6 months, assessment of BP at every visit with a goal systolic pressure less than $130 \mathrm{~mm} \mathrm{Hg}$, annual screening for microalbuminuria and retinopathy, and statin therapy for patients with clinical cardiovascular disease or age $\geq 40$ years, regardless of baseline lipid levels [19]. Previously published quality metrics data from a US population ( $\mathrm{n}=3131$ in Minnesota) showed similar $\mathrm{HbA}_{1 \mathrm{c}}$ of $7.3 \%$ and mean systolic $\mathrm{BP}$ of $133 \mathrm{~mm}$ $\mathrm{Hg}$, however, the rates of nephropathy and retinopathy 
Table 1. Quality of care measures in the Polish (Rzeszóvv) and American (Waukesha) cohorts

\begin{tabular}{lccc}
\hline Parameter & Rzeszów $\mathbf{n}=\mathbf{7 9}$ & Waukesha $\mathbf{n}=\mathbf{7 9}$ & P value \\
\hline $\mathrm{HbA}_{1 \mathrm{c}}(\%)$ mean $\pm \mathrm{SD}$ & $7.41 \pm 1.41$ & $8.04 \pm 2.14$ & 0.033 \\
Systolic blood pressure $[\mathrm{mm} \mathrm{Hg}]$ & $150.39 \pm 16.66$ & $131.99 \pm 16.71$ & $<0.001$ \\
Diastolic blood pressure $[\mathrm{mm} \mathrm{Hg}]$ & $80.62 \pm 11.51$ & $73.56 \pm 11.26$ & $<0.001$ \\
Statin usage (\%) & 90 & 86 & 0.450 \\
Nephropathy rate (\%) & 17 & 37 & 0.006 \\
Retinopathy rate (\%) & 10 & 21 & 0.069
\end{tabular}

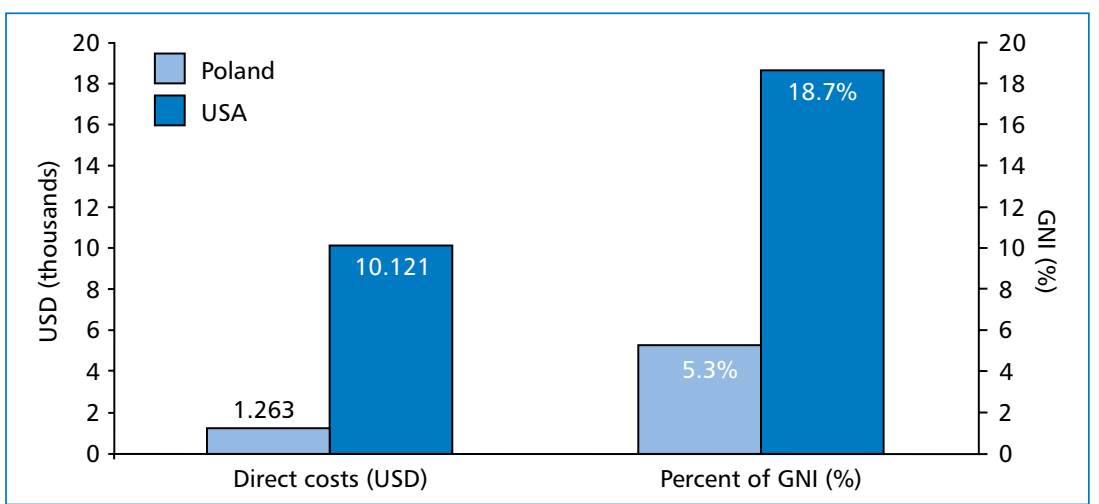

Figure 1. Direct medical costs of diabetes care in Rzeszów (Poland) and Waukesha (US) expressed in US dollars (USD) and as percent of Gross National Income (GNI) per capita

were significantly lower. Nephropathy and retinopathy rates in the cohort from Poland were significantly lower than in the US cohort [22]. The Wisconsin Collaborative for Healthcare Quality (WCHQ) reported 83\% statin usage at a clinic in Waukesha, WI which is consistent with our report in the US cohort [23]. A Polish study in 2013 described the quality of DM care for a 249-person cohort [24]. The mean $\mathrm{HbA}_{1 \mathrm{c}}$ was $7.3 \%$ and the mean systolic BP was $131 \mathrm{~mm} \mathrm{Hg}$. The rate of statin usage was $81 \%$. The rates of nephropathy and retinopathy were $31 \%$ and $42 \%$, respectively. While $\mathrm{HbA}_{1 \mathrm{c}}$ was comparable, BP level and statin usage were slightly higher in our Polish cohort compared to this previously published study. The rates of both nephropathy and retinopathy were both lower in our cohort.

The costs of care we estimated in our study are similar to previous studies in both countries. Our estimate of 1,263 USD for direct medical care in Poland is in line with previous estimates of approximately 1,000 USD in 2009 [24, 25]. Our estimate of 10,121 USD per year for direct medical care for patients with diabetes in the US is similar to previous estimates of approximately 10,000 USD per year in 2017 [6, 7]. Substitution of the estimated Medicare reimbursement for the actual reimbursement likely artificially lowered the US cost reported in this study.
Previous studies have either addressed cost or quality of diabetes care in isolation of each other, which makes it difficult to infer relationships between the quality of care and cost due to a paucity of controlled variables [13]. This is the first study to estimate both measures in the same study in populations matched for age, gender, BMI and socioeconomic status. Previous studies have used statistical methods to control for demographic variables. No other studies have utilized a matched cohort. Additionally, the similar quality results of our study are also unique in that it examines a chronic disease, instead of a particular procedure or episode of care. Examining a chronic disease that touches many different components of the health system using a matched cohort suggests that differences in cost are due to foundational differences in the health system. The results of our study suggest that the US health system delivers a similar quality of DM care at a significantly higher cost. Both groups were cared for by a sub-specialist. In addition, we attempted to control socioeconomic factors by matching for the city in which they primarily live in.

Burgeoning healthcare costs in the US threaten the fiscal sustainability of the country $[26,27]$. The high cost of healthcare in the US has been attributed to multiple reasons including wasteful spending, prescrip- 
tion drug costs, and advances in medical technology (technology creep). In addition, an aging workforce, unhealthy lifestyles, high administrative costs, lack of patient ownership of their care, and consolidation of provider practices all create an environment for unfettered cost growth [26, 27]. Previous debates have focused on delineating whether the high cost of healthcare in the US is due to high prices or high utilization $[12,28]$. This debate has by no means reached a consensus because many of these analyses focus on a single procedure or episode of care $[28,29]$. While our study did not valuate all of these factors, we did control for demographic features that can be considered as a higher risk for health care costs (i.e. older age, obesity, and care by a sub-specialist).

Our study is not free from several limitations. The first one is its retrospective nature and the exact study design was not able to be replicated in each region due to differences in health system infrastructure. In addition, the data cover the period 2013-2014, i.e. before the wider introduction to market newer drug classes: SGLT-2 (sodium-glucose cotransporter-2) inhibitors and GLP-1 (glucagon-like peptide-1) receptor agonists. Moreover, the tariffs for inpatient and outpatients services in Poland may not reflect the cost covered as in the US. In Poland, but also in the US, apart from the direct medical cost per patient covered by national payer or medical insurance services, substantial role can play patient's co-payment, especially for not-reimbursed or partly reimbursed drugs which depends on legal regulations and not necessarily on clinical practice. Despite we matched patients for gender, the decade of life and BMI to minimize bias, there are still many variables that could differ in each cohort, e.g. diabetes duration, presence of comorbidities, including cardiovascular disease (CVD) and the treatment used. We are aware that the list of the quality measures could be longer, but we were focused on the measures strictly associated with diabetes care i.e. glycemic control, blood pressure control, statin use and presence of microvascular complications. Macrovascular complications can develop independently of diabetes and frequently precede diabetes diagnosis, thus, we decided not to include this variable into quality measures. Additionally, another potential confounder is the severity of DM in each cohort. It is possible that primary care providers referred to less complex DM patients to an Endocrinologist in one of the cohorts. We were unable to evaluate other ADA recommendations such as annual dental care, and annual comprehensive foot exam. We also acknowledge that the political climate, patient and societal expectations, and litigation environments are mark- edly different between each country. Due to all these limitations, our findings cannot be generalized to the whole populations of both countries. Nevertheless, irrespective of these limitations, our study, conducted in comparable cohorts, indicate that similar diabetes control can be attained, in case of Poland, with substantially lower expenses.

\section{Conclusion}

The US continues to attempt healthcare reform in order to decrease cost and increase quality. This study reports minimal differences in quality and significant differences in cost between a matched cohort in Poland and the US and thus fills a critical gap in the literature by suggesting that gains in cost and quality may not be mutually exclusive. Further research is needed to understand how to best apply these lessons as health policy.

\section{Acknowledgments}

This project was supported by the National Center for Research Resources and the National Center for Advancing Translational Sciences, National Institutes of Health, through Grant Number UL1TR001436. Its contents are solely the responsibility of the authors and do not necessarily represent the official views of the NIH. This project is funded in part by the Advancing a Healthier Wisconsin endowment at the Medical College of Wisconsin. The authors wish to acknowledge the Decision Support staff at Froedtert Health and the Medical College of Wisconsin along with the staff of the Office of Clinical Research and Innovative Care Compliance at Froedtert Health. Additionally, the Dr. Elaine Kohler Summer Academy of Global Health Research for funding support of this project.

Part of these data was presented as a poster at the $78^{\text {th }}$ American Diabetes Association Scientific Sessions held in Orlando, 22-26 June 2018, and it was published as an abstract [Diabetes $2018 \mathrm{Jul} ; 67$ (Supplement 1): 1289-P. https://doi.org/10.2337/db18-1289-P].

\section{Conflict of interest}

The authors declare no conflict of interest.

\section{REFERENCES}

1. Davis K. Slowing the growth of health care costs - learning from international experience. N Engl J Med. 2008; 359(17): 1751-1755, doi: 10.1056/NEJMp0805261, indexed in Pubmed: 18946060

2. Shi L, Singh D. Delivering health care in America: a systems approach. Sixth edition. Jones \& Bartlett Learning, Burlington 2015.

3. Health resources - Health spending - OECD Data. Organization for Economic Cooperation and Development. http://data.oecd. org/healthres/health-spending.htm (19.04.2018). 
4. Health status - Life expectancy at birth - OECD Data. theOECD. http://data.oecd.org/healthstat/life-expectancy-at-birth. htm (19.04.2018).

5. Glass RI. What the United States has to gain from global health research. JAMA. 2013; 310(9): 903-904, doi: 10.1001/ jama.2013.276558, indexed in Pubmed: 24002270.

6. American Diabetes Association. Economic Costs of Diabetes in the U.S. in 2017. Diabetes Care. 2018; 41(5): 917-928, doi: 10.2337/ dci18-0007, indexed in Pubmed: 29567642.

7. Ozieh MN, Bishu KG, Dismuke CE, et al. Trends in health care expenditure in U.S. adults with diabetes: 2002-2011. Diabetes Care. 2015; 38(10): 1844-1851, doi: 10.2337/dc15-0369, indexed in Pubmed: 26203060.

8. Mata-Cases M, Casajuana M, Franch-Nadal J, et al. Direct medical costs attributable to type 2 diabetes mellitus: a populationbased study in Catalonia, Spain. Eur J Health Econ. 2016; 17(8): 1001-1010, doi: 10.1007/s10198-015-0742-5, indexed in Pubmed: 26542160.

9. Dawson KG, Gomes D, Gerstein $\mathrm{H}$, et al. The economic cost of diabetes in Canada, 1998. Diabetes Care. 2002; 25(8): 1303-1307, doi: 10.2337/diacare.25.8.1303, indexed in Pubmed: 12145225.

10. Köster I, Huppertz E, Hauner H, et al. Direct costs of diabetes mellitus in Germany - CoDiM 2000-2007. Exp Clin Endocrinol Diabetes. 2011; 119(6): 377-385, doi: 10.1055/s-0030-1269847, indexed in Pubmed: 21264804.

11. Anderson GF, Chalkidou K. Spending on medical care: more is better? JAMA. 2008; 299(20): 2444-2445, doi: 10.1001/ jama.299.20.2444, indexed in Pubmed: 18505956.

12. Dieleman JL, Squires $\mathrm{E}$, Bui $\mathrm{AL}$, et al. Factors associated with increases in US health care spending, 1996-2013. JAMA. 2017; 318(17): 1668-1678, doi: 10.1001/jama.2017.15927, indexed in Pubmed: 29114831.

13. Hussey PS, Wertheimer S, Mehrotra A. The association between health care quality and cost: a systematic review. Ann Intern Med. 2013; 158(1): 27-34, doi: 10.7326/0003-4819-158-1-20130101000006, indexed in Pubmed: 23277898.

14. About the Froedtert \& MCW Health Network | Froedtert \& the Medical College of Wis. https://www.froedtert.com/about (19.04.2019).

15. Information Service Rzeszow-Rzeszow in numbers. http://www rzeszow.pl/miasto-rzeszow/dane-statystyczne/rzeszow-w-liczbach (25.04.2018).
16. American FactFinder - Community Facts. U.S. Census Bureau. https://factfinder.census.gov/faces/nav/jsf/pages/community_facts. xhtml\# (25.04.2018)

17. Purchasing Power Parities for GDP and Related Indicators. OECD; 2018.

18. Vogel F. What Is a Purchasing Power Parity? World Bank 4.

19. American Diabetes Assocation. Standards of Medical Care in Diabetes. Diabetes Care. 2018; 41(Supplement 1).

20. Sagan A, Panteli D, Borkowski W, et al. Poland health system review. Health Syst Transit. 2011; 13(8): 1-193, indexed in Pubmed: 22551527.

21. Rogers A, Weiss ST. Epidemiologic and population genetic studies. Clinical and Translational Science. 2017: 313-326, doi: 10.1016/ b978-0-12-802101-9.00017-x.

22. Peterson KA, Radosevich DM, O'Connor PJ, et al. Improving Diabetes Care in Practice: findings from the TRANSLATE trial. Diabetes Care. 2008; 31 (12): 2238-2243, doi: 10.2337/dc08-2034, indexed in Pubmed: 18809622.

23. Diabetes: Statin Use Unless Contraindicated. Wisconsin Collaborative for Healthcare Quality; 2017.

24. Kudaj-Kurowska A, Turek I, Józefowska M, et al. [The metabolic control in type 2 diabetic patients according to Polish Diabetes Association recommendation]. Diabet Klin. 2014; 3 (3): 92-99.

25. Leśniowska J, Schubert $A$, Wojna $M$, et al. Costs of diabetes and its complications in Poland. Eur J Health Econ. 2014; 15(6): 653-660, doi: 10.1007/s10198-014-0644-y.

26. Orszag PR, Ellis P. Addressing rising health care costs - a view from the Congressional Budget Office. N Engl J Med. 2007; 357(19): 1885-1887, doi: 10.1056/NEJMp078191, indexed in Pubmed: 17989379.

27. Chernew ME, Baicker K, Hsu J. The specter of financial Armageddon - health care and federal debt in the United States. N Engl J Med. 2010; 362(13): 1166-1168, doi: 10.1056/NEJMp1002873, indexed in Pubmed: 20237338.

28. Spiro T, Lee EO, Emanuel EJ. Price and utilization: why we must target both to curb health care costs. Ann Intern Med. 2012; 157(8): 586-590, doi: 10.7326/0003-4819-157-8-20121016000014, indexed in Pubmed: 23070492.

29. Bodenheimer T, Fernandez A. High and rising health care costs. Part 4: can costs be controlled while preserving quality? Ann Intern Med. 2005; 143(1): 26-31, doi: 10.7326/0003-4819-1431-200507050-00007, indexed in Pubmed: 15998752. 